\title{
Supplementary Appendix to: Stock Return and Cash Flow Predictability: The Role of Volatility Risk
}

\author{
Tim Bollerslev, Lai Xu, and Hao Zhou ${ }^{\ddagger}$
}

September 16, 2013

*Department of Economics, Duke University, Durham NC 27708 USA, and NBER and CREATES, boller@econ.duke.edu, 919-660-1846.

${ }^{\dagger}$ Department of Economics, Duke University, Durham NC 27708 USA, lai.xu@ duke.edu, 919-257-0059.

‡PBC School of Finance, Tsinghua University, Beijing, 100083, P. R. China, zhouh@pbcsf.tsinghua.edu.cn, 1062790655 


\section{A Model Solution}

Our basic solution method for the model is adopted from Bansal and Yaron (2004), Bansal, Kiku, and Yaron (2007), and Drechsler and Yaron (2011). To begin, we follow Campbell and Shiller (1988) and solve for the return on consumption by log-linearizing $r_{c, t+1}$ around the unconditional mean of the wealth-consumption ratio $v_{t}$,

$$
r_{c, t+1} \approx \kappa_{0}+\kappa_{1} v_{t+1}-v_{t}+\Delta c_{t+1}
$$

where $\kappa_{1}=\frac{\exp (E(v))}{1+\exp (E(v))}$, and $\kappa_{0}=\log [1+\exp (E(v))]-\kappa_{1} E(v)$. We then conjecture a solution for $v_{t}$ as a linear function of the state vector $Y_{t}$,

$$
v_{t}=A_{0}+A^{\prime} Y_{t},
$$

where $A_{0}$ is a scalar, and $A=\left(0, A_{x}, A_{\sigma}, A_{q}, 0\right)$ refer to the pricing coefficients. Next, by substituting $v_{t}$ and $v_{t+1}$ into equation (A.1), both $r_{c, t+1}$ and the stochastic discount factor $m_{t+1}$ defined in the main text may be expressed as linear functions of the state vector,

$$
\begin{aligned}
& m_{t+1}=\mu_{m}-\left(\gamma e_{1}^{\prime}+(1-\theta) \kappa_{1} A^{\prime}\right) Y_{t+1}-(\theta-1) A^{\prime} Y_{t}, \\
& r_{c, t+1}=\mu_{r_{c}}+\left(e_{1}^{\prime}+\kappa_{1} A^{\prime}\right) Y_{t+1}-A^{\prime} Y_{t} .
\end{aligned}
$$

Going one step further, it follows that the innovations to the pricing kernel and the return on the wealth claim may be expressed as,

$$
\begin{aligned}
m_{t+1}-E_{t}\left(m_{t+1}\right) & =-\Lambda^{\prime} H G_{t} z_{t+1}, \\
r_{c, t+1}-E_{t}\left(r_{c, t+1}\right) & =\Lambda_{c}^{\prime} H G_{t} z_{t+1},
\end{aligned}
$$

where $\Lambda$ denotes the price of risk for the factor shocks,

$$
\Lambda=\gamma e_{1}+\kappa_{1}(1-\theta) A,
$$

for $e_{1} \equiv[1,0,0,0,0]$, and $\Lambda_{c}=e_{1}+\kappa_{1} A$. The magnitude and sign of $\Lambda$ are determined by the preference parameter $\theta$ and the pricing coefficient vector $A$. If investors prefer early resolution of uncertainty, i.e., $\gamma>\phi^{-1}$, $A$ reveals the sensitivity of the market prices for the different shocks to higher order consumption dynamics. When $\gamma=\phi^{-1}$ (CRRA case), $\Lambda$ collapses to $\gamma e_{1}$, and only transient shocks to consumption growth level $z_{g, t+1}$ are priced.

Since the no-arbitrage condition must hold regardless of the realization of the state vector $Y_{t}$, it is possible to solve for $A$ by imposing the Euler equation,

$$
0=\mu_{m}+\mu_{r_{c}}+\left[\left(-\Lambda+\Lambda_{c}\right)^{\prime} F-\theta A^{\prime}\right] Y_{t}+\frac{1}{2}\left(-\Lambda+\Lambda_{c}\right)^{\prime} H G_{t} G_{t}^{\prime} H^{\prime}\left(-\Lambda+\Lambda_{c}\right) .
$$


This in turn implies that

$$
\begin{aligned}
\theta A_{\{i\}}+\left(\tilde{\Lambda}_{c}^{\prime} F\right)_{\{i\}} & =\frac{1}{2}\left[1_{i=3} \sum_{j=1,5}\left(\tilde{\Lambda}_{c}^{\prime} h_{j}\right)^{2}+1_{i=4} \sum_{j=2,3,4}\left(\tilde{\Lambda}_{c}^{\prime} h_{j}\right)^{2}\right], \\
0 & =\mu_{m}+\mu_{r_{c}},
\end{aligned}
$$

where $\tilde{\Lambda}_{c}=-\Lambda_{c}+\Lambda=(\gamma-1) e_{1}-\kappa_{1} \theta A, i$ refers to the $i^{t h}$ element of vector, and $1_{i=n}$ is an indicator function. The solutions are,

$$
\begin{aligned}
& A_{x}=-\frac{\gamma-1}{\theta\left(1-\kappa_{1} \rho_{x}\right)}, \\
& A_{\sigma}=\frac{(\gamma-1)^{2}}{2 \theta\left(1-\kappa_{1} \rho_{\sigma}\right)},
\end{aligned}
$$

while $A_{q}$ solves the equation $\frac{1}{2} a_{q} \theta^{2} A_{q}^{2}+\left(b_{q}+\left(1-\kappa_{1} \rho_{q}\right)\right)\left(-\theta A_{q}\right)+\frac{1}{2} c_{q}=0$, where

$$
\begin{aligned}
& a_{q}=\kappa_{1}^{2}\left(\varphi_{x}^{2} s_{q, x}^{2}+s_{q, \sigma}^{2}+\varphi_{q}^{2}\right)>0, \\
& b_{q}=\kappa_{1}^{2}\left(\varphi_{x}^{2}\left(-A_{x} \theta-A_{\sigma} \theta s_{\sigma, x}\right) s_{q, x}-A_{\sigma} \theta s_{q, \sigma}\right), \\
& c_{q}=\kappa_{1}^{2}\left(\varphi_{x}^{2}\left(-A_{x} \theta-A_{\sigma} \theta s_{\sigma, x}\right)^{2}+A_{\sigma}^{2} \theta^{2}\right)>0 .
\end{aligned}
$$

Since $a_{q}>0$ and $c_{q}>0$, the two roots are either negative or positive. We choose the larger root for $-\theta A_{q}$ if $b_{q}+\left(1-\kappa_{1} \rho_{q}\right)>0$, or the smaller root if $b_{q}+\left(1-\kappa_{1} \rho_{q}\right)<0$. In both cases $A_{q}$ reduces to zero when $s_{q, x}, s_{q, \sigma}$ and $\varphi_{q}$ are zero.

Even though no closed-form expressions for $A$ are available when we consider $\kappa_{0}$ and $\kappa_{1}$ as endogenous, the system of equations are still solvable. As shown in equation (A.8), $A$ depends on $\kappa_{1}, \mu, F, H$, as well as the preference parameters. Considering the definitions of $\kappa_{1}$ and $\kappa_{0}, \kappa_{1}$ and $A$ are the only unknowns in the constant term in the Euler equation, so that $\kappa_{1}$ may be solved endogenously together with $A$. Finally, $\kappa_{0}$ and $A_{0}$ can be expressed as functions of $A$ and $\kappa_{1}$. For detailed numerical solutions, see Drechsler and Yaron (2011) Appendix A.1 and A.2.

Applying a similar conjecture-evaluation type method, it is possible to solve for the aggregate market return $r_{t, t+1}$. Denote the price-dividend ratio by $w_{t}$, and consider the conjecture solution $w_{t}=A_{d, 0}+A_{d}^{\prime} Y_{t}$. Log-linearize $r_{t, t+1}$ around the unconditional mean of the price-dividend ratio yields,

$$
r_{t, t+1} \approx \kappa_{d, 0}+\kappa_{d, 1} w_{t+1}-w_{t}+\Delta d_{t+1} .
$$

Substituting out $w_{t}$ and $w_{t+1}$ in the above equation, the return on the market may be rewritten as,

$$
r_{t, t+1}=\mu_{r_{d}}+\left(e_{5}^{\prime}+\kappa_{d, 1} A_{d}^{\prime}\right) Y_{t+1}-A_{d}^{\prime} Y_{t}
$$

where $\Lambda_{d}=e_{5}+\kappa_{d, 1} A_{d}$ and $A_{d}=\left[0, A_{d, x}, A_{d, \sigma}, A_{d, q}, A_{d, d}\right]^{\prime}$ is a vector of pricing coefficients. 
Using the same solution method as the one previously used for $A$, it follows by the no-arbitrage condition,

$$
0=\mu_{m}+\mu_{r_{d}}+\left[\left(-\Lambda+\Lambda_{d}\right)^{\prime} F-(\theta-1) A^{\prime}-A_{d}^{\prime}\right]^{\prime} Y_{t}+0.5\left(-\Lambda+\Lambda_{d}\right)^{\prime} H G_{t} G_{t}^{\prime} H^{\prime}\left(-\Lambda+\Lambda_{d}\right),
$$

which implies that

$$
\begin{aligned}
(\theta-1) A_{\{i\}}+A_{d,\{i\}}+\left(\tilde{\Lambda}_{d}^{\prime} F\right)_{\{i\}} & =0.5\left[1_{i=3} \sum_{j=1,5}\left(\tilde{\Lambda}_{d}^{\prime} h_{j}\right)^{2}+1_{i=4} \sum_{j=2,3,4}\left(\tilde{\Lambda}_{d}^{\prime} h_{j}\right)^{2}\right], \\
0 & =\mu_{m}+\mu_{r_{d}},
\end{aligned}
$$

where $\tilde{\Lambda}_{d}=-\Lambda_{d}+\Lambda=\gamma e_{1}-e_{5}+\kappa_{1}(1-\theta) A-\kappa_{d, 1} A_{d}$.

The solution for $A_{d}$ may therefore be expressed as,

$$
\begin{aligned}
A_{d, d} & =\frac{\rho_{d}}{1-\kappa_{d, 1} \rho_{d}} \\
\frac{1-\kappa_{1} \rho_{x}}{1-\kappa_{d, 1} \rho_{x}}(1-\theta) A_{x}-A_{d, x} & =-\frac{-\gamma+\phi_{d x}\left(1+\kappa_{d, 1} A_{d, d}\right)}{1-\kappa_{d, 1} \rho_{x}} \\
\frac{1-\kappa_{1} \rho_{\sigma}}{1-\kappa_{d, 1} \rho_{\sigma}}(1-\theta) A_{\sigma}-A_{d, \sigma} & =-\frac{1}{2} \frac{\gamma^{2}+\varphi_{d}^{2}\left(1+\kappa_{d, 1} A_{d, d}\right)^{2}}{1-\kappa_{d, 1} \rho_{\sigma}}<0 \\
\frac{1-\kappa_{1} \rho_{q}}{1-\kappa_{d, 1} \rho_{q}}(1-\theta) A_{q}-A_{d, q} & =-\frac{1}{2} \frac{a_{d, q}\left(\kappa_{1}(1-\theta) A_{q}-\kappa_{d, 1} A_{d, q}\right)^{2}+2 b_{d, q}\left(\kappa_{1}(1-\theta) A_{q}-\kappa_{d, 1} A_{d, q}\right)+c_{d, q}}{1-\kappa_{d, 1} \rho_{q}}
\end{aligned}
$$

where

$$
\begin{aligned}
a_{d, q}= & a_{q}=\left(\varphi_{x}^{2} s_{q, x}^{2}+s_{q, \sigma}^{2}+\varphi_{q}^{2}\right)>0 \\
b_{d, q}= & \left(\varphi_{x}^{2}\left(\kappa_{1}(1-\theta) A_{x}-\kappa_{d, 1} A_{d, x}+\left(\kappa_{1}(1-\theta) A_{\sigma}-\kappa_{d, 1} A_{d, \sigma}\right) s_{\sigma, x}+\frac{-1}{1-\kappa_{d, 1} \rho_{d}} s_{d, x}\right) s_{q, x}\right) \\
& +\left(\left(\kappa_{1}(1-\theta) A_{\sigma}-\kappa_{d, 1} A_{d, \sigma}+\frac{-1}{1-\kappa_{d, 1} \rho_{d}} s_{d, \sigma}\right) s_{q, \sigma}+\frac{-1}{1-\kappa_{d, 1} \rho_{d}} s_{d, q} \varphi_{q}^{2}\right) \\
c_{d, q}= & \left(\varphi_{x}^{2}\left(\kappa_{1}(1-\theta) A_{x}-\kappa_{d, 1} A_{d, x}+\left(\kappa_{1}(1-\theta) A_{\sigma}-\kappa_{d, 1} A_{d, \sigma}\right) s_{\sigma, x}+\frac{-1}{1-\kappa_{d, 1} \rho_{d}} s_{d, x}\right)^{2}\right) \\
& +\left(\left(\kappa_{1}(1-\theta) A_{\sigma}-\kappa_{d, 1} A_{d, \sigma}+\frac{-1}{1-\kappa_{d, 1} \rho_{d}} s_{d, \sigma}\right)^{2}+\left(\frac{-1}{1-\kappa_{d, 1} \rho_{d}} s_{d, q}\right)^{2} \varphi_{q}^{2}\right)>0
\end{aligned}
$$

In other words, $A_{d, q}$ solves the equation (A.20) and we choose the root with smaller absolute value. Both $A_{q}$ and $A_{d, q}$ reduce to zero when $s_{q, x}, s_{q, \sigma}$ and $\varphi_{q}$ are all zero. We will discuss the sign of $A_{d, q}$ later on in the parameter implication section. We can explicitly express $A_{d, x}$ and $A_{d, \sigma}$ as

$$
\begin{aligned}
& A_{d, x}=\frac{(1-\gamma) / \theta-1+\phi_{d x} /\left(1-\kappa_{d, 1} \rho_{d}\right)}{1-\kappa_{d, 1} \rho_{x}}=\frac{-\psi^{-1}+\phi_{d x} /\left(1-\kappa_{d, 1} \rho_{d}\right)}{1-\kappa_{d, 1} \rho_{x}} \\
& A_{d, \sigma}=\frac{(\gamma-1)^{2}+2 \theta \gamma+\theta\left(\varphi_{d}^{2} /\left(1-\kappa_{d, 1} \rho_{d}\right)^{2}-1\right)}{2 \theta\left(1-\kappa_{d, 1} \rho_{\sigma}\right)} .
\end{aligned}
$$




\section{B Variance Risk Premium}

In order to determine the factor structure for the variance risk premium, we first need to solve for the second order moment of the return $r_{t, t+1}$. It follows from above that $r_{t, t+1}-E_{t}\left(r_{t, t+1}\right)=\Lambda_{d}^{\prime} H G_{t} z_{t+1}$, so that the conditional variance of the return is affine in $\sigma_{t}^{2}$ and $q_{t}$,

$$
\begin{aligned}
\operatorname{Var}_{t}\left(r_{t, t+1}\right) & =\sum_{j=1,5} \Lambda_{d}^{\prime} h_{j} h_{j}^{\prime} \Lambda_{d} \sigma_{t}^{2}+\sum_{j=2,3,4} \Lambda_{d}^{\prime} h_{j} h_{j}^{\prime} \Lambda_{d} q_{t} \\
& =\left(1+\kappa_{d, 1} A_{d, d}\right)^{2} \varphi_{d}^{2} \sigma_{t}^{2}+\sum_{j=2,3,4} \Lambda_{d}^{\prime} h_{j} h_{j}^{\prime} \Lambda_{d} q_{t} .
\end{aligned}
$$

The first term is associated with the volatility of cash flow shocks, and the second term represents the consumption uncertainty. Accordingly, the equity risk premium may be expressed as,

$$
\begin{aligned}
\log \left(E_{t} R_{t, t+1}\right)-r_{f, t} & =E_{t}\left(r_{t, t+1}\right)+\frac{1}{2} \operatorname{Var}_{t}\left(r_{t, t+1}\right)-r_{f, t} \\
& =-\operatorname{Cov}_{t}\left(m_{t+1}, r_{t, t+1}\right)=\sum_{j=2,3,4} \Lambda_{d}^{\prime} h_{j} h_{j}^{\prime} \Lambda q_{t}
\end{aligned}
$$

The first equality comes from the normality distribution of $r_{t, t+1} \equiv \log \left(R_{t, t+1}\right)$, the second equality comes from the no arbitrage condition and $r_{f, t}=\log \left(E_{t} m_{t+1}+\frac{1}{2} \operatorname{Var}_{t}\left(m_{t+1}\right)\right)$. The expectations of $\operatorname{Var}_{t}\left(r_{t, t+1}\right)$ under the physical and risk-neutral probability measures are,

$$
\begin{aligned}
& E_{t}\left(\operatorname{Var}_{t+1}\left(r_{d, t+2}\right)\right)=\sum_{j=1,5} \Lambda_{d}^{\prime} h_{j} h_{j}^{\prime} \Lambda_{d}\left(\mu_{\sigma}+\rho_{\sigma} \sigma_{t}^{2}\right)+\sum_{j=2,3,4} \Lambda_{d}^{\prime} h_{j} h_{j}^{\prime} \Lambda_{d}\left(\mu_{q}+\rho_{q} q_{t}\right), \\
& E_{t}^{Q}\left(\operatorname{Var}_{t+1}\left(r_{d, t+2}\right)\right)=\sum_{j=1,5} \Lambda_{d}^{\prime} h_{j} h_{j}^{\prime} \Lambda_{d}\left(\mu_{\sigma}+\rho_{\sigma} \sigma_{t}^{2}+s_{q, 1} q_{t}\right)+\sum_{j=2,3,4} \Lambda_{d}^{\prime} h_{j} h_{j}^{\prime} \Lambda_{d}\left(\mu_{q}+\rho_{q} q_{t}+s_{q, 2} q_{t}\right) .
\end{aligned}
$$

Under the risk neutral measure, we reweight probabilities according to the pricing kernel $\frac{e^{m_{t, t+1}}}{E_{t} e^{m_{t, t+1}}}$. If investor prefers early resolution of uncertainty, the shocks $z_{t+1}$ 's conditional mean shifts away from zero. And this shift can be expressed as the conditional covariance between the state vector and SDF $m_{t, t+1}$,

$$
\begin{aligned}
& s_{q, 1} q_{t}=\operatorname{Cov}_{t}\left(e_{3} H G_{t} z_{t+1},-\Lambda^{\prime} H G_{t} z_{t+1}\right) \\
& =\quad-\left(\varphi_{x} s_{\sigma, x} h_{2}^{\prime}+h_{3}^{\prime}\right) \Lambda q_{t}, \\
& s_{q, 2} q_{t}=\operatorname{Cov}_{t}\left(e_{4} H G_{t} z_{t+1},-\Lambda^{\prime} H G_{t} z_{t+1}\right) \\
& =-\left(\varphi_{x} s_{q, x} h_{2}^{\prime}+s_{q, \sigma} h_{3}^{\prime}+\varphi_{q} h_{4}^{\prime}\right) \Lambda q_{t},
\end{aligned}
$$


where,

$$
\begin{aligned}
s_{q, 1} & =-\kappa_{1}(1-\theta)\left(A_{x} \varphi_{x}^{2} s_{\sigma, x}+A_{\sigma}\left(\varphi_{x}^{2} s_{\sigma, x}^{2}+1\right)+A_{q}\left(\varphi_{x}^{2} s_{\sigma, x} s_{q, x}+s_{q, \sigma}\right)\right) \\
& =-\kappa_{1}(1-\theta)\left(\varphi_{x}^{2} s_{\sigma, x} s_{q, x}+s_{q, \sigma}\right)\left(\frac{A_{x} \varphi_{x}^{2} s_{\sigma, x}+A_{\sigma}\left(\varphi_{x}^{2} s_{\sigma, x}^{2}+1\right)}{\varphi_{x}^{2} s_{\sigma, x} s_{q, x}+s_{q, \sigma}}+A_{q}\right) \\
s_{q, 2} & =-\kappa_{1}(1-\theta)\left(A_{x} \varphi_{x}^{2} s_{q, x}+A_{\sigma}\left(\varphi_{x}^{2} s_{\sigma, x} s_{q, x}+s_{q, \sigma}\right)+A_{q}\left(\varphi_{x}^{2} s_{q, x}^{2}+s_{q, \sigma}^{2}+\varphi_{q}^{2}\right)\right) \\
& =-1 / \kappa_{1}(1-\theta) a_{q}\left(\frac{b_{q}}{-\theta a_{q}}+A_{q}\right)
\end{aligned}
$$

By definition, $s_{q, 1}$ and $s_{q, 2}$ represent the market prices of shocks to $\sigma_{t}^{2}$ and $q_{t}$, respectively. Thus, the variance risk premium is naturally defined by,

$$
\begin{aligned}
V R P_{t} & \equiv \quad E_{t}^{Q}\left(\operatorname{Var}_{t+1}\left(r_{d, t+2}\right)\right)-E_{t}\left(\operatorname{Var}_{t+1}\left(r_{d, t+2}\right)\right) \\
& =\left(\sum_{j=1,5} \Lambda_{d}^{\prime} h_{j} h_{j}^{\prime} \Lambda_{d} s_{q, 1}+\sum_{j=2,3,4} \Lambda_{d}^{\prime} h_{j} h_{j}^{\prime} \Lambda_{d} s_{q, 2}\right) q_{t}
\end{aligned}
$$

In the main text, we will refer to the expected return variation and the variance risk premium as,

$$
\begin{aligned}
& E R V_{t}=\frac{Q_{1,1}}{\rho_{\sigma}}\left(\mu_{\sigma}+\rho_{\sigma} \sigma_{t}^{2}\right)+\frac{Q_{1,2}}{\rho_{q}}\left(\mu_{q}+\rho_{q} q_{t}\right), \\
& V R P_{t}=Q_{2,2} q_{t}
\end{aligned}
$$

for short, where

$$
\begin{array}{lc}
Q_{1,1}= & \sum_{j=1,5} \Lambda_{d}^{\prime} h_{j} h_{j}^{\prime} \Lambda_{d} \rho_{\sigma}>0, \\
Q_{1,2}= & \sum_{j=2,3,4} \Lambda_{d}^{\prime} h_{j} h_{j}^{\prime} \Lambda_{d} \rho_{q}>0, \\
Q_{2,2}= & \frac{Q_{1,1}}{\rho_{\sigma}} s_{q, 1}+\frac{Q_{1,2}}{\rho_{q}} s_{q, 2} .
\end{array}
$$

In order to determine the signs of $A_{d, x}, A_{d, \sigma}$ and $A_{d, q}$, it is informative to write out the formula in terms of the estimated $B$ and $\tilde{\rho}$ matrices,

$$
\frac{\phi_{d, x}}{-A_{d, x}}=\tilde{\rho}_{3,4}, \quad \frac{A_{d, \sigma}}{Q_{1,1}}=B_{4,1}, \quad \frac{Q_{1,2}}{Q_{2,2}}=-B_{1,2}, \quad \frac{A_{d, q}}{Q_{2,2}}=B_{4,2}-B_{1,2} B_{4,1} .
$$

Since $\tilde{\rho}_{3,4}<0, \phi_{d, x}$ and $A_{d, x}$ must have the same signs. Thus, by definition $Q_{1,1}>0$ and $Q_{1,2}>0$, which together with the estimates for $B_{4,1}=-0.60<0$ and $B_{1,2}=-0.02<0$, imply that $A_{d, \sigma}<0$ and $Q_{2,2}>0$. Consequently $A_{d, q}=Q_{2,2}\left(B_{4,2}-B_{1,2} B_{4,1}\right)=-1.45 Q_{2,2}<0$. 


\section{Alternative Setups}

\section{C.1 Separate Volatility Processes}

We will consider the following alternative setup for $G_{t}$ and $H$, with $F$ unchanged,

$$
G_{t}=\left(\begin{array}{ccccc}
\sigma_{t} & 0 & 0 & 0 & 0 \\
0 & \sqrt{q_{t}} & 0 & 0 & 0 \\
0 & 0 & \sigma_{t} & 0 & 0 \\
0 & 0 & 0 & \sqrt{q_{t}} & 0 \\
0 & 0 & 0 & 0 & \sigma_{t}
\end{array}\right) \quad H=\left(\begin{array}{ccccc}
1 & 0 & 0 & 0 & 0 \\
0 & 1 & 0 & 0 & 0 \\
0 & s_{\sigma, x} & \varphi_{\sigma} & 0 & 0 \\
0 & s_{q, x} & 0 & \varphi_{q} & 0 \\
0 & s_{d, x} & \varphi_{\sigma} s_{d, \sigma} & \varphi_{q} s_{d, q} & \varphi_{d}
\end{array}\right)
$$

This setup is related to Bansal and Shaliastovich (2013), where the volatilities of $x_{t}$ and $\sigma_{t}^{2}$ are modeled as two separate processes.

For simplicity, we use the same general notation as in the main setup for $A, \Lambda, \Lambda_{c}$ and $\Lambda_{d}$. However, the solutions for the pricing coefficients are obviously different from the main setup, except for $A_{d, d}=\frac{\rho_{d}}{1-\kappa_{d, 1} \rho_{d}}$,

$$
\begin{aligned}
\theta A_{\{i\}}+\left(\tilde{\Lambda}_{c}^{\prime} F\right)_{\{i\}} & =0.5\left[1_{i=3} \sum_{j=1,3,5}\left(\tilde{\Lambda}_{c}^{\prime} h_{j}\right)^{2}+1_{i=4} \sum_{j=2,4}\left(\tilde{\Lambda}_{c}^{\prime} h_{j}\right)^{2}\right] \\
(\theta-1) A_{\{i\}}+A_{d,\{i\}}+\left(\tilde{\Lambda}_{d}^{\prime} F\right)_{\{i\}} & =0.5\left[1_{i=3} \sum_{j=1,3,5}\left(\tilde{\Lambda}_{d}^{\prime} h_{j}\right)^{2}+1_{i=4} \sum_{j=2,4}\left(\tilde{\Lambda}_{d}^{\prime} h_{j}\right)^{2}\right] .
\end{aligned}
$$

Since $r_{t, t+1}-E_{t}\left(r_{t, t+1}\right)=\Lambda_{d}^{\prime} H G_{t} z_{t+1}$, the conditional variance of the return is again affine,

$$
\operatorname{Var}_{t}\left(r_{t, t+1}\right)=\sum_{j=1,3,5} \Lambda_{d}^{\prime} h_{j} h_{j}^{\prime} \Lambda_{d} \sigma_{t}^{2}+\sum_{j=2,4} \Lambda_{d}^{\prime} h_{j} h_{j}^{\prime} \Lambda_{d} q_{t}
$$

The expectations of $\operatorname{Var}_{t}\left(r_{t, t+1}\right)$ under the physical and risk-neutral probability measures may further be expressed as,

$$
\begin{aligned}
& E_{t}\left(\operatorname{Var}_{t+1}\left(r_{d, t+2}\right)\right)=\sum_{j=1,3,5} \Lambda_{d}^{\prime} h_{j} h_{j}^{\prime} \Lambda_{d}\left(\mu_{\sigma}+\rho_{\sigma} \sigma_{t}^{2}\right)+\sum_{j=2,4} \Lambda_{d}^{\prime} h_{j} h_{j}^{\prime} \Lambda_{d}\left(\mu_{q}+\rho_{q} q_{t}\right) \\
& E_{t}^{Q}\left(\operatorname{Var}_{t+1}\left(r_{d, t+2}\right)\right)=\sum_{j=1,3,5} \Lambda_{d}^{\prime} h_{j} h_{j}^{\prime} \Lambda_{d}\left(\mu_{\sigma}+\rho_{\sigma} \sigma_{t}^{2}+s_{q, 1} q_{t}+s_{\sigma, 1} \sigma_{t}^{2}\right)+\sum_{j=2,4} \Lambda_{d}^{\prime} h_{j} h_{j}^{\prime} \Lambda_{d}\left(\mu_{q}+\rho_{q} q_{t}+s_{q, 2} q_{t}\right)
\end{aligned}
$$

If investors prefer early resolution of uncertainty, the conditional means of the $z_{t+1}$ shocks shift away from zero under the risk-neutral measure,

$$
\begin{aligned}
s_{\sigma, 1} \sigma_{t}^{2}+s_{q, 1} q_{t} & =\operatorname{Cov}_{t}\left(e_{3}^{\prime} H G_{t} z_{t+1},-\Lambda^{\prime} H G_{t} z_{t+1}\right) \\
& =-\varphi_{\sigma} h_{3}^{\prime} \Lambda \sigma_{t}^{2}-\left(\varphi_{x} s_{\sigma, x} h_{2}^{\prime}\right) \Lambda q_{t}, \\
s_{q, 2} q_{t} & =\operatorname{Cov}_{t}\left(e_{4} H G_{t} z_{t+1},-\Lambda^{\prime} H G_{t} z_{t+1}\right) \\
& =-\left(\varphi_{x} s_{q, x} h_{2}^{\prime}+\varphi_{q} h_{4}^{\prime}\right) \Lambda q_{t} .
\end{aligned}
$$


Defining the variance risk premium as before,

$$
\begin{aligned}
V R P_{t} & \equiv E_{t}^{Q}\left(\operatorname{Var}_{t+1}\left(r_{d, t+2}\right)\right)-E_{t}\left(\operatorname{Var}_{t+1}\left(r_{d, t+2}\right)\right) \\
& =\sum_{j=1,3,5} \Lambda_{d}^{\prime} h_{j} h_{j}^{\prime} \Lambda_{d}\left(s_{\sigma, 1} \sigma_{t}^{2}+s_{q, 1} q_{t}\right)+\sum_{j=2,4} \Lambda_{d}^{\prime} h_{j} h_{j}^{\prime} \Lambda_{d} s_{q, 2} q_{t},
\end{aligned}
$$

we may express the expected return variation and premium in short-hand form as,

$$
\begin{aligned}
& E R V_{t}=\frac{Q_{1,1}}{\rho_{\sigma}}\left(\mu_{\sigma}+\rho_{\sigma} \sigma_{t}^{2}\right)+\frac{Q_{1,2}}{\rho_{q}}\left(\mu_{q}+\rho_{q} q_{t}\right), \\
& V R P_{t}=Q_{2,1} \sigma_{t}^{2}+Q_{2,2} q_{t},
\end{aligned}
$$

where

$$
\begin{array}{ll}
Q_{1,1}=\sum_{j=1,3,5} \Lambda_{d}^{\prime} h_{j} h_{j}^{\prime} \Lambda_{d} \rho_{\sigma}>0 & Q_{1,2}=\sum_{j=2,4} \Lambda_{d}^{\prime} h_{j} h_{j}^{\prime} \Lambda_{d} \rho_{q}>0 \\
Q_{2,1}=\frac{Q_{1,1}}{\rho_{q}} s_{\sigma, 1} & Q_{2,2}=\frac{Q_{1,1}}{\rho_{\sigma}} s_{q, 1}+\frac{Q_{1,2}}{\rho_{q}} s_{q, 2} .
\end{array}
$$

\section{C.2 Long-Run Stochastic Volatility}

We will consider the following alternative setup for $G_{t}, H$, and $F$,

$$
F=\left(\begin{array}{ccccc}
0 & 1 & 0 & 0 & 0 \\
0 & \rho_{x} & 0 & 0 & 0 \\
0 & 0 & \rho_{\sigma} & 1 & 0 \\
0 & 0 & 0 & \rho_{q} & 0 \\
0 & \phi_{d x} & 0 & 0 & \rho_{d}
\end{array}\right) \quad G_{t}=\left(\begin{array}{ccccc}
\sigma_{t} & 0 & 0 & 0 & 0 \\
0 & \sigma_{t} & 0 & 0 & 0 \\
0 & 0 & \sigma_{t} & 0 & 0 \\
0 & 0 & 0 & \sqrt{q_{t}} & 0 \\
0 & 0 & 0 & 0 & \sigma_{t}
\end{array}\right) \quad H=\left(\begin{array}{cccccc}
1 & 0 & 0 & 0 & 0 \\
0 & \varphi_{x} & 0 & 0 & 0 \\
0 & \varphi_{x} s_{\sigma, x} & \varphi_{\sigma} & 0 & 0 \\
0 & \varphi_{x} s_{q, x} & 0 & \varphi_{q} & 0 \\
0 & \varphi_{x} s_{d, x} & \varphi_{\sigma} s_{d, \sigma} & \varphi_{q} s_{d, q} & \varphi_{d}
\end{array}\right)
$$

This setup is motivated by the model analyzed by Branger and Vòlkert (2012), among others, allowing for a time-varying mean of the consumption variance $\sigma_{t}^{2}$.

Again, for simplicity we will use the same general notation as in the main setup for $A, \Lambda, \Lambda_{c}$ and $\Lambda_{d}$. The solution for $A_{d, d}=\frac{\rho_{d}}{1-\kappa_{d, 1} \rho_{d}}$ remains the same, but the other the pricing coefficients now take the form,

$$
\begin{aligned}
\theta A_{\{i\}}+\left(\tilde{\Lambda}_{c}^{\prime} F\right)_{\{i\}} & =\frac{1}{2}\left[1_{i=3} \sum_{j=1,2,3,5}\left(\tilde{\Lambda}_{c}^{\prime} h_{j}\right)^{2}+1_{i=4} \sum_{j=4}\left(\tilde{\Lambda_{c}^{\prime}} h_{j}\right)^{2}\right], \\
(\theta-1) A_{\{i\}}+A_{d,\{i\}}+\left(\tilde{\Lambda}_{d}^{\prime} F\right)_{\{i\}} & =\frac{1}{2}\left[1_{i=3} \sum_{j=1,2,3,5}\left(\tilde{\Lambda}_{d}^{\prime} h_{j}\right)^{2}+1_{i=4} \sum_{j=4}\left(\tilde{\Lambda_{d}^{\prime}} h_{j}\right)^{2}\right] .
\end{aligned}
$$

As before, $r_{t, t+1}-E_{t}\left(r_{t, t+1}\right)=\Lambda_{d}^{\prime} H G_{t} z_{t+1}$, so that the conditional variance of the return may be expressed as,

$$
\operatorname{Var}_{t}\left(r_{t, t+1}\right)=\sum_{j=1,2,3,5} \Lambda_{d}^{\prime} h_{j} h_{j}^{\prime} \Lambda_{d} \sigma_{t}^{2}+\sum_{j=4} \Lambda_{d}^{\prime} h_{j} h_{j}^{\prime} \Lambda_{d} q_{t}
$$


The expectation of $\operatorname{Var}_{t}\left(r_{t, t+1}\right)$ under the physical and risk-neutral probability measures are,

$$
\begin{aligned}
& E_{t}\left(\operatorname{Var}_{t+1}\left(r_{d, t+2}\right)\right)=\sum_{j=1,2,3,5} \Lambda_{d}^{\prime} h_{j} h_{j}^{\prime} \Lambda_{d}\left(\mu_{\sigma}+\rho_{\sigma} \sigma_{t}^{2}+q_{t}\right)+\sum_{j=4} \Lambda_{d}^{\prime} h_{j} h_{j}^{\prime} \Lambda_{d}\left(\mu_{q}+\rho_{q} q_{t}\right), \\
& E_{t}^{Q}\left(\operatorname{Var}_{t+1}\left(r_{d, t+2}\right)\right)=\sum_{j=1,2,3,5} \Lambda_{d}^{\prime} h_{j} h_{j}^{\prime} \Lambda_{d}\left(\mu_{\sigma}+\rho_{\sigma} \sigma_{t}^{2}+q_{t}+s_{\sigma, 1} \sigma_{t}^{2}\right)+\sum_{j=4} \Lambda_{d}^{\prime} h_{j} h_{j}^{\prime} \Lambda_{d}\left(\mu_{q}+\rho_{q} q_{t}+s_{\sigma, 2} \sigma_{t}^{2}+s_{q, 2} q_{t}\right) .
\end{aligned}
$$

The shifts in the conditional means of the $z_{t+1}$ shocks under the risk-neutral measure become,

$$
\begin{aligned}
s_{\sigma, 1} \sigma_{t}^{2} & =\operatorname{Cov}_{t}\left(e_{3}^{\prime} H G_{t} z_{t+1},-\Lambda^{\prime} H G_{t} z_{t+1}\right) \\
& =-\varphi_{\sigma} h_{3}^{\prime} \Lambda \sigma_{t}^{2}-\left(\varphi_{x} s_{\sigma, x} h_{2}^{\prime}\right) \Lambda \sigma_{t}^{2}, \\
s_{\sigma, 2} \sigma_{t}^{2}+s_{q, 2} q_{t} & =\operatorname{Cov}_{t}\left(e_{4} H G_{t} z_{t+1},-\Lambda^{\prime} H G_{t} z_{t+1}\right) \\
& =-\left(\varphi_{x} s_{q, x} h_{2}^{\prime}\right) \Lambda \sigma_{t}^{2}-\left(\varphi_{q} h_{4}^{\prime}\right) \Lambda q_{t} .
\end{aligned}
$$

As before, the expected return variation and variance risk premium, may be conveniently expressed as,

$$
\begin{aligned}
& E R V_{t}=\frac{Q_{1,1}}{\rho_{\sigma}}\left(\mu_{\sigma}+\rho_{\sigma} \sigma_{t}^{2}\right)+\frac{Q_{1,2}}{\rho_{q}}\left(\mu_{q}+\rho_{q} q_{t}\right), \\
& V R P_{t}=Q_{2,1} \sigma_{t}^{2}+Q_{2,2} q_{t},
\end{aligned}
$$

where

$$
\begin{array}{ll}
Q_{1,1}=\sum_{j=1,2,3,5} \Lambda_{d}^{\prime} h_{j} h_{j}^{\prime} \Lambda_{d} \rho_{\sigma}>0 & Q_{1,2}=\sum_{j=4} \Lambda_{d} h_{j} h_{j}^{\prime} \Lambda_{d} \rho_{q}>0 \\
Q_{2,1}=\frac{Q_{1,1}}{\rho_{\sigma}} s_{\sigma, 1}+\frac{Q_{1,2}}{\rho_{q}} s_{\sigma, 2} & Q_{2,2}=\frac{Q_{1,2}}{\rho_{q}} s_{q, 2} .
\end{array}
$$




\section{Detailed Derivations for Section 2.2}

Substituting $f_{t}$ by $Q^{-1}\left(X_{t}-\mu_{X}\right)$ in the basic relation $f_{t+1}=\mu+\rho f_{t}+S \epsilon_{t+1}$, it follows that

$$
Q^{-1} X_{t+1}=\mu+Q^{-1} \mu_{X}-\rho Q^{-1} \mu_{X}+\rho Q^{-1} X_{t}+S \epsilon_{t+1} .
$$

Normalizing each element of $Q^{-1} X_{t+1}$ by the corresponding diagonal element of $Q^{-1}$, the model may be rewritten as,

$$
B X_{t+1}=\tilde{\mu}+\tilde{\rho} B X_{t}+\tilde{S} \tilde{\epsilon}_{t+1}
$$

where

$$
B \equiv\left(\frac{1}{\operatorname{diag}\left(Q^{-1}\right)} \otimes l_{1 \times 4}\right) \odot Q^{-1}
$$

To match with equation (D.49),

$$
\tilde{\mu}=\left(\frac{1}{\operatorname{diag}\left(Q^{-1}\right)} \otimes l_{1 \times 4}\right) \odot\left(\mu-\rho Q^{-1} \mu_{X}\right),
$$

and

$$
\begin{aligned}
\tilde{\rho} & =\left[\left(\frac{1}{\operatorname{diag}\left(Q^{-1}\right)} \otimes l_{1 \times 4}\right) \odot\left(\rho Q^{-1}\right)\right] B^{-1} \\
& =\left[\left(\frac{1}{\operatorname{diag}\left(Q^{-1}\right)} \otimes l_{1 \times 4}\right) \odot\left(Q^{-1} \operatorname{diag}(\rho)+(\rho-\operatorname{diag}(\rho)) Q^{-1}\right)\right] B^{-1} \\
& =\left[\left(\frac{1}{\operatorname{diag}\left(Q^{-1}\right)} \otimes l_{1 \times 4}\right) \odot\left(Q^{-1} \odot\left(\operatorname{vec}(\operatorname{diag}(\rho)) \otimes l_{1 \times 4}\right)+(\rho-\operatorname{diag}(\rho)) Q^{-1}\right)\right] B^{-1} \\
& =\left[B \odot\left(\operatorname{vec}(\operatorname{diag}(\rho)) \otimes l_{1 \times 4}+\frac{\rho-\operatorname{diag}(\rho)}{-A_{d, x}} B\right] B^{-1}\right. \\
& =\rho+\frac{\rho-\operatorname{diag}(\rho)}{-A_{d, x}}
\end{aligned}
$$

or

Defining $\tilde{\epsilon}_{t+1}$ as

$$
\tilde{\epsilon}_{t+1} \equiv \frac{1}{\operatorname{diag}\left(Q^{-1}\right)} \odot \epsilon_{t+1},
$$

it follows again from equation (D.49) that

$$
\tilde{S}=\left(\frac{1}{\operatorname{diag}\left(Q^{-1}\right)} \otimes l_{1 \times 4}\right) \odot S \odot \frac{1}{\frac{1}{\operatorname{diag}_{\left(Q^{-1}\right)^{\prime}}} \otimes l_{1 \times 4}} .
$$

Based on the formula for $Q$ in the main text, the inverse $Q^{-1}$ and $\frac{1}{\operatorname{diag}\left(Q^{-1}\right)}$ may be expressed as,

$$
Q^{-1}=\left(\begin{array}{cccc}
\frac{1}{Q_{1,1}} & \frac{-Q_{1,2}}{Q_{1,1} Q_{2,2}} & 0 & 0 \\
0 & \frac{1}{Q_{2,2}} & 0 & 0 \\
0 & 0 & 1 & 0 \\
\frac{-A_{d, \sigma}}{Q_{1,1} A_{d, x}} & \frac{-Q_{1,1} A_{d, q}+Q_{1,2} A_{d, \sigma}}{Q_{1,1} Q_{2,2} A_{d, x}} & \frac{-A_{d, d}}{A_{d, x}} & -\frac{1}{A_{d, x}}
\end{array}\right) \quad \frac{1}{\operatorname{diag}\left(Q^{-1}\right)}=\left(\begin{array}{c}
Q_{1,1} \\
Q_{2,2} \\
1 \\
-A_{d, x}
\end{array}\right)
$$


Combining the expressions for $\rho$ and $S$, it therefore follows that

$$
\begin{gathered}
B=\left(\begin{array}{cccc}
1 & -\frac{Q_{1,2}}{Q_{2,2}} & 0 & 0 \\
0 & 1 & 0 & 0 \\
0 & 0 & 1 & 0 \\
\frac{A_{d, \sigma}}{Q_{1,1}} & \frac{Q_{1,1} A_{d, q}-A_{d, \sigma} Q_{1,2}}{Q_{1,1} Q_{2,2}} & A_{d, d} & 1
\end{array}\right) \quad \tilde{\rho}=\left(\begin{array}{cccc}
\rho_{\sigma} & 0 & 0 & 0 \\
0 & \rho_{q} & 0 & 0 \\
0 & 0 & \rho_{d} & \frac{\phi_{d x}}{-A_{d, x}} \\
0 & 0 & 0 & \rho_{x}
\end{array}\right) \\
\tilde{S}=\left(\begin{array}{cccc}
1 & 0 & 0 & \frac{Q_{1,1}}{-A_{d, x}} s_{\sigma, x} \\
\frac{Q_{2,2}}{Q_{1,1}} s_{q, \sigma} & 1 & 0 & \frac{Q_{2,2}}{-A_{d, x}} s_{q, x} \\
\frac{1}{Q_{1,1}} s_{d, \sigma} & \frac{1}{Q_{2,2}} s_{d, q} & 1 & \frac{1}{-A_{d, x}} s_{d, x} \\
0 & 0 & 0 & 1
\end{array}\right) \quad \tilde{\epsilon}_{t+1}=\left(\begin{array}{c}
Q_{1,1} \\
Q_{2,2} \\
1 \\
-A_{d, x}
\end{array}\right) \odot \epsilon_{t+1} .
\end{gathered}
$$

\section{D.1 Separate Volatility Dynamics}

In the alternative setup with separate volatility dynamic, $\rho, \epsilon_{t+1}$ and $\mathrm{S}$ may be expressed as,

$$
\begin{gathered}
\rho=\left(\begin{array}{cccc}
\rho_{\sigma} & 0 & 0 & 0 \\
0 & \rho_{q} & 0 & 0 \\
0 & 0 & \rho_{d} & \phi_{d x} \\
0 & 0 & 0 & \rho_{x}
\end{array}\right) \quad \epsilon_{t+1}=\left(\begin{array}{c}
\varphi_{\sigma} \sigma_{t} z_{\sigma, t+1} \\
\varphi_{q} \sqrt{q_{t}} z_{q, t+1} \\
\varphi_{d} \sigma_{t} z_{d, t+1} \\
\sqrt{q_{t}} z_{x, t+1}
\end{array}\right) \quad S=\left(\begin{array}{cccc}
1 & 0 & 0 & s_{\sigma, x} \\
0 & 1 & 0 & s_{q, x} \\
s_{d, \sigma} & s_{d, q} & 1 & s_{d, x} \\
0 & 0 & 0 & 1
\end{array}\right) \\
X_{t}=\mu_{X}+Q f_{t} \quad Q=\left(\begin{array}{cccc}
Q_{1,1} & Q_{1,2} & 0 & 0 \\
Q_{2,1} & Q_{2,2} & 0 & 0 \\
0 & 0 & 1 & 0 \\
-A_{d, \sigma} & -A_{d, q} & -A_{d, d} & -A_{d, x}
\end{array}\right)
\end{gathered}
$$

Consequently,

$$
Q^{-1}=\left(\begin{array}{cccc}
\frac{Q_{2,2}}{Q_{1,1} Q_{2,2}-Q_{1,2} Q_{2,1}} & \frac{-Q_{1,2}}{Q_{1,1} Q_{2,2}-Q_{1,2} Q_{2,1}} & 0 & 0 \\
\frac{Q_{1,1}}{Q_{1,1} Q_{2,2}-Q_{1,2} Q_{2,1}} & \frac{Q_{1,1} Q_{2,2}-Q_{1,2} Q_{2,1}}{0} & 0 & 0 \\
0 & 0 & 1 & 0 \\
\frac{Q_{2,1} A_{d, q}-Q_{2,2} A_{d, \sigma}}{\left(Q_{1,1} Q_{2,2}-Q_{1,2} Q_{2,1}\right) A_{d, x}} & \frac{-Q_{1,1} A_{d, q}+Q_{1,2} A_{d, \sigma}}{\left(Q_{1,1} Q_{2,2}-Q_{1,2} Q_{2,1}\right) A_{d, x}} & \frac{-A_{d, d}}{A_{d, x}} & -\frac{1}{A_{d, x}}
\end{array}\right) \quad \frac{1}{\operatorname{diag}\left(Q^{-1}\right)}=\left(\begin{array}{c}
\frac{Q_{1,1} Q_{2,2}-Q_{1,2} Q_{2,1}}{Q_{2,2}} \\
\frac{Q_{1,1} Q_{2,2}-Q_{1,2} Q_{2,1}}{Q_{1,1}} \\
1 \\
-A_{d, x}
\end{array}\right)
$$

Combining these expressions, it follows that

$$
\begin{gathered}
B=\left(\begin{array}{ccccc}
1 & \frac{-Q_{1,2}}{Q_{2,2}} & 0 & 0 \\
\frac{-Q_{2,1}}{Q_{1,1}} & 1 & 0 & 0 \\
0 & 0 & 1 & 0 \\
\frac{-Q_{2,1} A_{d, q}+Q_{2,2} A_{d, \sigma}}{Q_{1,1} Q_{2,2}-Q_{1,2} Q_{2,1}} & \frac{+Q_{1,1} A_{d, q}-Q_{1,2} A_{d, \sigma}}{Q_{1,1} Q_{2,2}-Q_{1,2} Q_{2,1}} & A_{d, d} & 1
\end{array}\right) \quad \tilde{\rho}=\left(\begin{array}{cccc}
\rho_{\sigma} & 0 & 0 & 0 \\
0 & \rho_{q} & 0 & 0 \\
0 & 0 & \rho_{d} & \frac{\phi_{d x}}{-A_{d, x}} \\
0 & 0 & 0 & \rho_{x}
\end{array}\right) \\
\tilde{S}=\left(\begin{array}{cccc}
1 & 0 & \frac{Q_{1,1} Q_{2,2}-Q_{1,2} Q_{2,1}}{-Q_{2,2} A_{d, x}} s_{\sigma, x} \\
0 & 1 & 0 & \frac{Q_{1,1} Q_{2,2}-Q_{1,2} Q_{2,1}}{-Q_{1,1} A_{d, x}} s_{q, x} \\
\frac{Q_{2,2}}{Q_{1,1} Q_{2,2}-Q_{1,2} Q_{2,1}} s_{d, \sigma} & \frac{Q_{1,1}}{Q_{1,1} Q_{2,2}-Q_{1,2} Q_{2,1}} s_{d, q} & 1 & \frac{1}{-A_{d, x}} s_{d, x} \\
0 & 0 & 0 & 1
\end{array}\right) \tilde{\epsilon}_{t+1}=\left(\begin{array}{c}
\frac{Q_{1,1} Q_{2,2}-Q_{1,2} Q_{2,1}}{Q_{2,2}} \\
\frac{Q_{1,1} Q_{2,2}-Q_{1,2} Q_{2,1}}{Q_{1,1}} \\
1 \\
-A_{d, x}
\end{array}\right) \odot \epsilon_{t+1} .
\end{gathered}
$$




\section{D.2 Stochastic Volatility in the Long-Run}

In the alternative setup with stochastic volatility in the long-run drift, $\rho, \epsilon_{t+1}$ and $\mathrm{S}$ may be expressed as,

$$
\begin{gathered}
\rho=\left(\begin{array}{cccc}
\rho_{\sigma} & 1 & 0 & 0 \\
0 & \rho_{q} & 0 & 0 \\
0 & 0 & \rho_{d} & \phi_{d x} \\
0 & 0 & 0 & \rho_{x}
\end{array}\right) \epsilon_{t+1}=\left(\begin{array}{c}
\varphi_{\sigma} \sigma_{t} z_{\sigma, t+1} \\
\varphi_{q} \sqrt{q_{t}} z_{q, t+1} \\
\varphi_{d} \sigma_{t} z_{d, t+1} \\
\varphi_{x} \sigma_{t} z_{x, t+1}
\end{array}\right) \quad S=\left(\begin{array}{cccc}
1 & 0 & 0 & s_{\sigma, x} \\
0 & 1 & 0 & s_{q, x} \\
s_{d, \sigma} & s_{d, q} & 1 & s_{d, x} \\
0 & 0 & 0 & 1
\end{array}\right) \\
X_{t}=\mu_{X}+Q f_{t} \quad Q=\left(\begin{array}{cccc}
Q_{1,1} & Q_{1,2} & 0 & 0 \\
Q_{2,1} & Q_{2,2} & 0 & 0 \\
0 & 0 & 1 & 0 \\
-A_{d, \sigma} & -A_{d, q} & -A_{d, d} & -A_{d, x}
\end{array}\right)
\end{gathered}
$$

Consequently,

$$
Q^{-1}=\left(\begin{array}{cccc}
\frac{Q_{2,2}}{Q_{1,1} Q_{2,2}-Q_{1,2} Q_{2,1}} & \frac{-Q_{1,2}}{Q_{1,1} Q_{2,2}-Q_{1,2} Q_{2,1}} & 0 & 0 \\
\frac{Q_{1,1}}{Q_{1,1} Q_{2,2}-Q_{1,2} Q_{2,1}} & \frac{0}{Q_{1,1} Q_{2,2}-Q_{1,2} Q_{2,1}} & 0 & 0 \\
0 & 0 & 1 & 0 \\
\frac{Q_{2,1} A_{d, q}-Q_{2,2} A_{d, \sigma}}{\left(Q_{1,1} Q_{2,2}-Q_{1,2} Q_{2,1}\right) A_{d, x}} & \frac{-Q_{1,1} A_{d, q}+Q_{1,2} A_{d, \sigma}}{\left(Q_{1,1} Q_{2,2}-Q_{1,2} Q_{2,1}\right) A_{d, x}} & \frac{-A_{d, d}}{A_{d, x}} & -\frac{1}{A_{d, x}}
\end{array}\right) \quad \frac{1}{\operatorname{diag}\left(Q^{-1}\right)}=\left(\begin{array}{c}
\frac{Q_{1,1} Q_{2,2}-Q_{1,2} Q_{2,1}}{Q_{2,2}} \\
\frac{Q_{1,1} Q_{2,2}-Q_{1,2} Q_{2,1}}{Q_{1,1}} \\
1 \\
-A_{d, x}
\end{array}\right)
$$

Combining these expressions, it follows that

$$
\begin{gathered}
B=\left(\begin{array}{ccccc}
1 & \frac{-Q_{1,2}}{Q_{2,2}} & 0 & 0 \\
\frac{-Q_{2,1}}{Q_{1,1}} & 1 & 0 & 0 \\
0 & 0 & 1 & 0 \\
\frac{-Q_{2,1} A_{d, q}+Q_{2,2} A_{d, \sigma}}{Q_{1,1} Q_{2,2}-Q_{1,2} Q_{2,1}} & \frac{+Q_{1,1} A_{d, q}-Q_{1,2} A_{d, \sigma}}{Q_{1,1} Q_{2,2}-Q_{1,2} Q_{2,1}} & A_{d, d} & 1
\end{array}\right) \quad \tilde{\rho}=\left(\begin{array}{cccc}
\rho_{\sigma} & \frac{Q_{1,1}}{Q_{2,2}} & 0 & 0 \\
0 & \rho_{q} & 0 & 0 \\
0 & 0 & \rho_{d} & \frac{\phi_{d x}}{-A_{d, x}} \\
0 & 0 & 0 & \rho_{x}
\end{array}\right) \\
\tilde{S}=\left(\begin{array}{cccc}
1 & 0 & 0 & \frac{Q_{1,1} Q_{2,2}-Q_{1,2} Q_{2,1}}{-Q_{2,2} A_{d, x}} s_{\sigma, x} \\
0 & 1 & 0 & \frac{Q_{1,1} Q_{2,2}-Q_{1,2} Q_{2,1}}{-Q_{1,1} A_{d, x}} s_{q, x} \\
\frac{Q_{1,2}}{Q_{2,1}-Q_{1,2} Q_{2,1}} s_{d, \sigma} & \frac{1}{Q_{1,1} Q_{2,2}-Q_{1,2} Q_{2,1}} s_{d, q} & 1 & \frac{1}{-A_{d, x}} s_{d, x} \\
0 & 0 & 0 & 1
\end{array}\right) \quad \tilde{\epsilon}_{t+1}=\left(\begin{array}{c}
\frac{Q_{1,1} Q_{2,2}-Q_{1,2} Q_{2,1}}{Q_{2,2}} \\
\frac{Q_{1,1} Q_{2,2}-Q_{1,2} Q_{2,1}}{Q_{1,1}} \\
1 \\
-A_{d, x}
\end{array}\right) \odot \epsilon_{t+1} .
\end{gathered}
$$




\section{Table D.1 Structural Factor GARCH Estimates-Separate Volatility Dynamics}

The table reports the "structural" factor GARCH estimates for the alternative setup with separate volatility dynamics described in Sections C.1 and D.1, with the three restrictions: $A_{d, d}=\frac{\rho_{d}}{1-\kappa_{d, 1} \rho_{d}}, \Gamma_{4,4}+\Upsilon_{4,4}=\rho_{q}$, and $\Gamma_{3,3}=0$. The resulting $J$-test with 7 degrees-of-freedom for the GMM-based estimation equals 26.31 , corresponding to a p-value 0.0004 .

\begin{tabular}{|c|c|c|c|c|c|c|c|c|c|c|}
\hline$B$ & & & $E R V_{t+1}$ & & $V R P_{t+1}$ & & $\Delta d_{t+1}$ & & $d_{t+1} / p_{t+1}$ & \\
\hline$E R V_{t+1}$ & & & 1 & & -0.490 & $(0.117)$ & 0 & & 0 & \\
\hline$V R P_{t+1}$ & & & -0.022 & $(0.030)$ & 1 & & 0 & & 0 & \\
\hline$\Delta d_{t+1}$ & & & 0 & & 0 & & 1 & & 0 & \\
\hline$d_{t+1} / p_{t+1}$ & & & -0.110 & $(0.141)$ & -1.595 & $(0.063)$ & -0.158 & & 1 & \\
\hline$\tilde{\rho}$ & constant & & $E R V_{t}$ & & $V R P_{t}$ & & $\Delta d_{t}$ & & $d_{t} / p_{t}$ & \\
\hline$E R V_{t+1}$ & 0.009 & $(0.003)$ & 0.827 & $(0.089)$ & 0 & & 0 & & 0 & \\
\hline$V R P_{t+1}$ & 0.008 & $(0.002)$ & 0 & & 0.312 & $(0.071)$ & 0 & & 0 & \\
\hline$\Delta d_{t+1}$ & -0.002 & $(0.016)$ & 0 & & 0 & & -0.187 & $(0.035)$ & -0.001 & ( 0.004$)$ \\
\hline$d_{t+1} / p_{t+1}$ & -0.080 & $(0.029)$ & 0 & & 0 & & 0 & & 0.980 & $(0.008)$ \\
\hline$\tilde{S}$ & & & $\tilde{\boldsymbol{\epsilon}}_{\sigma_{t+1}^{2}}$ & & $\tilde{\epsilon}_{q_{t+1}}$ & & $\tilde{\epsilon}_{\Delta d_{t+1}}$ & & $\tilde{\epsilon}_{x, t+1}$ & \\
\hline$E R V_{t+1}$ & & & 1 & & 0 & & 0 & & 0.316 & $(0.038)$ \\
\hline$V R P_{t+1}$ & & & 0 & & 1 & & 0 & & -0.245 & $(0.017)$ \\
\hline$\Delta d_{t+1}$ & & & -0.387 & $(0.080)$ & -0.134 & $(0.160)$ & 1 & & 0.095 & $(0.034)$ \\
\hline$d_{t+1} / p_{t+1}$ & & & 0 & & 0 & & 0 & & 1 & \\
\hline$\tilde{\epsilon}$ & $\varpi_{u}$ & & $\Gamma$ & & $\Upsilon$ & & & & & \\
\hline$E R V_{t+1}$ & 0.001 & $(0.000)$ & 0.153 & $(0.360)$ & 0.388 & $(0.138)$ & & & & \\
\hline$V R P_{t+1}$ & 0.000 & $(0.000)$ & 0.537 & $(0.335)$ & 0.116 & ( 0.079) & & & & \\
\hline$\Delta d_{t+1}$ & 0.001 & $(0.000)$ & 0 & & 0.449 & $(0.115)$ & & & & \\
\hline$d_{t+1} / p_{t+1}$ & 0.002 & $(0.000)$ & 0.167 & & 0.144 & $(0.106)$ & & & & \\
\hline
\end{tabular}

Table D.2 Structural Model Implications-Separate Volatility Dynamics

The table reports the contemporaneous matrix $\Phi_{0}$, the reduced form matrix $\Phi$, and the return equation, implied by the alternative "structural" factor GARCH model defined in Sections C.1 and D.1).

\begin{tabular}{|c|c|c|c|c|c|c|c|c|c|c|}
\hline$\Phi_{0}^{-1} \equiv B^{-1} \tilde{S}$ & & & $E R V_{t+1}$ & & $V R P_{t+1}$ & & $\Delta d_{t+1}$ & & $d_{t+1} / p_{t+1}$ & \\
\hline$E R V_{t+1}$ & & & 1.011 & $(0.015)$ & 0.496 & $(0.118)$ & 0 & & 0.198 & $(0.049)$ \\
\hline$V R P_{t+1}$ & & & 0.022 & $(0.030)$ & 1 & & 0 & & -0.241 & $(0.017)$ \\
\hline$\Delta d_{t+1}$ & & & -0.387 & $(0.080)$ & -0.134 & $(0.160)$ & 1 & & 0.095 & $(0.034)$ \\
\hline$d_{t+1} / p_{t+1}$ & & & 0.085 & $(0.134)$ & 1.646 & $(0.079)$ & 0.158 & $(0.025)$ & 0.652 & $(0.047)$ \\
\hline$\Phi \equiv B^{-1} \tilde{\rho} B$ & constant & & $E R V_{t}$ & & $V R P_{t}$ & & $\Delta d_{t}$ & & $d_{t} / p_{t}$ & \\
\hline$E R V_{t+1}$ & 0.013 & $(0.003)$ & 0.833 & $(0.091)$ & -0.255 & $(0.075)$ & 0 & & 0 & \\
\hline$V R P_{t+1}$ & 0.008 & $(0.001)$ & 0.012 & $(0.016)$ & 0.306 & $(0.070)$ & 0.000 & $(0.000)$ & 0.000 & $(0.000)$ \\
\hline$\Delta d_{t+1}$ & -0.002 & $(0.016)$ & 0.000 & $(0.000)$ & 0.002 & $(0.006)$ & -0.187 & $(0.035)$ & -0.001 & $(0.004)$ \\
\hline$d_{t+1} / p_{t+1}$ & -0.066 & $(0.030)$ & 0.002 & $(0.045)$ & -1.103 & $(0.130)$ & -0.185 & $(0.035)$ & 0.980 & $(0.008)$ \\
\hline & \multicolumn{10}{|c|}{ GMM Implied Return Equation } \\
\hline \multirow[t]{3}{*}{$r_{t, t+1}$} & constant & & $E R V_{t}$ & & $V R P_{t}$ & & $\Delta d_{t}$ & & $d_{t} / p_{t}$ & \\
\hline & 0.062 & $(0.029)$ & -0.002 & $(0.044)$ & 1.074 & $(0.127)$ & -0.007 & $(0.002)$ & 0.046 & $(0.009)$ \\
\hline & & & $\tilde{\epsilon}_{\sigma_{t+1}^{2}}$ & & $\tilde{\epsilon}_{q_{t+1}}$ & & $\tilde{\epsilon}_{\Delta d_{t+1}}$ & & $\tilde{\epsilon}_{x, t+1}$ & \\
\hline stru-shocks & & & -0.470 & $(0.213)$ & -1.734 & $(0.178)$ & 0.846 & $(0.041)$ & -0.539 & $(0.036)$ \\
\hline
\end{tabular}




\section{Table D.3 Structural Factor GARCH Estimates-Long-Run Stochastic Volatility}

The table reports the "structural" factor GARCH estimates for the alternative setup with long-run stochastic volatility described in Sections C. 2 and D.2, with the two restrictions: $A_{d, d}=\frac{\rho_{d}}{1-K_{d, 1} \rho_{d}}$ and $\Gamma_{3,3}=0$. The resulting $J$-test with 6 degrees-of-freedom for the GMM-based estimation equals 37.02 , corresponding to a p-value 0.0000 .

\begin{tabular}{|c|c|c|c|c|c|c|c|c|c|c|}
\hline B & & & $E R V_{t+1}$ & & $V R P_{t+1}$ & & $\Delta d_{t+1}$ & & $d_{t+1} / p_{t+1}$ & \\
\hline$E R V_{t+1}$ & & & 1 & & 0.000 & $(0.189)$ & 0 & & 0 & \\
\hline$V R P_{t+1}$ & & & 0.120 & $(0.039)$ & 1 & & 0 & & 0 & \\
\hline$\Delta d_{t+1}$ & & & 0 & & 0 & & 1 & & 0 & \\
\hline$d_{t+1} / p_{t+1}$ & & & -0.016 & $(0.086)$ & -2.007 & $(0.156)$ & -0.249 & & 1 & \\
\hline$\tilde{\rho}$ & constant & & $E R V_{t}$ & & $V R P_{t}$ & & $\Delta d_{t}$ & & $d_{t} / p_{t}$ & \\
\hline$E R V_{t+1}$ & 0.003 & $(0.002)$ & 1.001 & $(0.077)$ & -0.070 & $(0.255)$ & 0 & & 0 & \\
\hline$V R P_{t+1}$ & 0.006 & $(0.001)$ & 0 & & 0.609 & $(0.079)$ & 0 & & 0 & \\
\hline$\Delta d_{t+1}$ & -0.000 & $(0.014)$ & 0 & & 0 & & -0.329 & $(0.040)$ & -0.001 & $(0.004)$ \\
\hline$d_{t+1} / p_{t+1}$ & -0.075 & $(0.028)$ & 0 & & 0 & & 0 & & 0.982 & $(0.007)$ \\
\hline$\tilde{S}$ & & & $\tilde{\epsilon}_{\sigma_{t+1}^{2}}$ & & $\tilde{\epsilon}_{q_{t+1}}$ & & $\tilde{\epsilon}_{\Delta d_{t+1}}$ & & $\tilde{\epsilon}_{x, t+1}$ & \\
\hline$E R V_{t+1}$ & & & 1 & & 0 & & 0 & & 0.332 & $(0.036)$ \\
\hline$V R P_{t+1}$ & & & 0 & & 1 & & 0 & & -0.186 & $(0.016)$ \\
\hline$\Delta d_{t+1}$ & & & -0.524 & $(0.061)$ & -0.069 & $(0.147)$ & 1 & & 0.061 & $(0.024)$ \\
\hline$d_{t+1} / p_{t+1}$ & & & 0 & & 0 & & 0 & & 1 & \\
\hline$\tilde{\epsilon}$ & $\varpi_{u}$ & & $\Gamma$ & & $\Upsilon$ & & & & & \\
\hline$E R V_{t+1}$ & 0.001 & $(0.000)$ & 0.001 & $(0.051)$ & 0.776 & $(0.062)$ & & & & \\
\hline$V R P_{t+1}$ & 0.000 & $(0.000)$ & 0.000 & $(0.139)$ & 0.322 & $(0.147)$ & & & & \\
\hline$\Delta d_{t+1}$ & 0.001 & $(0.000)$ & 0 & & 0.454 & $(0.095)$ & & & & \\
\hline$d_{t+1} / p_{t+1}$ & 0.002 & $(0.000)$ & 0.766 & $(0.061)$ & 0.160 & $(0.041)$ & & & & \\
\hline
\end{tabular}

\section{Table D.4 Structural Model Implications-Long-Run Stochastic Volatility}

The table reports the contemporaneous matrix $\Phi_{0}$, the reduced form matrix $\Phi$, and the return equation, implied by the alternative "structural" factor GARCH model in Sections C.2 and D.2.

\begin{tabular}{|c|c|c|c|c|c|c|c|c|c|c|}
\hline$\Phi_{0}^{-1} \equiv B^{-1} \tilde{S}$ & & & $E R V_{t+1}$ & & $V R P_{t+1}$ & & $\Delta d_{t+1}$ & & $d_{t+1} / p_{t+1}$ & \\
\hline$E R V_{t+1}$ & & & 1.000 & $(0.023)$ & -0.000 & $(0.189)$ & 0 & & 0.332 & $(0.032)$ \\
\hline$V R P_{t+1}$ & & & -0.120 & $(0.037)$ & 1 & & 0 & & -0.226 & $(0.025)$ \\
\hline$\Delta d_{t+1}$ & & & -0.524 & $(0.061)$ & -0.069 & $(0.147)$ & 1 & & 0.061 & $(0.024)$ \\
\hline$d_{t+1} / p_{t+1}$ & & & -0.356 & $(0.052)$ & 1.990 & $(0.158)$ & 0.249 & $(0.023)$ & 0.568 & $(0.051)$ \\
\hline$\Phi \equiv B^{-1} \tilde{\rho} B$ & constant & & $E R V_{t}$ & & $V R P_{t}$ & & $\Delta d_{t}$ & & $d_{t} / p_{t}$ & \\
\hline$E R V_{t+1}$ & 0.003 & $(0.002)$ & 0.993 & $(0.089)$ & -0.070 & $(0.194)$ & 0 & & 0 & \\
\hline$V R P_{t+1}$ & 0.005 & $(0.001)$ & -0.046 & $(0.020)$ & 0.618 & ( 0.069$)$ & 0 & & 0 & \\
\hline$\Delta d_{t+1}$ & -0.000 & $(0.014)$ & 0.000 & $(0.000)$ & 0.003 & $(0.007)$ & -0.328 & $(0.040)$ & -0.001 & ( 0.004$)$ \\
\hline$d_{t+1} / p_{t+1}$ & -0.065 & $(0.029)$ & -0.093 & $(0.041)$ & -0.732 & $(0.174)$ & -0.326 & $(0.041)$ & 0.982 & $(0.007)$ \\
\hline & \multicolumn{10}{|c|}{ GMM Implied Return Equation } \\
\hline \multirow[t]{3}{*}{$r_{t, t+1}$} & constant & & $E R V_{t}$ & & $V R P_{t}$ & & $\Delta d_{t}$ & & $d_{t} / p_{t}$ & \\
\hline & 0.063 & $(0.027)$ & 0.090 & $(0.040)$ & 0.714 & $(0.169)$ & -0.011 & $(0.002)$ & 0.045 & $(0.008)$ \\
\hline & & & $\tilde{\epsilon}_{\sigma_{t+1}^{2}}$ & & $\tilde{\epsilon}_{q_{t+1}}$ & & $\tilde{\epsilon}_{\Delta d_{t+1}}$ & & $\tilde{\epsilon}_{x, t+1}$ & \\
\hline stru-shocks & & & -0.178 & $(0.124)$ & -2.004 & $(0.181)$ & 0.758 & $(0.050)$ & -0.491 & $(0.035)$ \\
\hline
\end{tabular}




\section{References}

Bansal, Ravi, Dana Kiku, and Amir Yaron (2007), "A Note on the Economics and Statistics of Predictability: A Long Run Risks Perspective," Working Paper, Duke University and University of Pennsylvania.

Bansal, Ravi and Ivan Shaliastovich (2013), "A Long-Run Risks Explanation of Preditability Puzzles in Bond and Currency Markets," Review of Financial Studies, vol. 26, 1-33.

Bansal, Ravi and Amir Yaron (2004), "Risks for the Long Run: A Potential Resolution of Asset Pricing Puzzles," Journal of Finance, vol. 59, 1481-1509.

Branger, Nicole and Clemens Vòlkert (2012), "What is the Equilibrium Price of Variance Risk? A Long-Run Risk Model with Two Volatility Factors," Working Paper, University of Muenster.

Campbell, John Y. and Robert J. Shiller (1988), "Stock Prices, Earnings, and Expected Dividends," Journal of Finance, vol. 43, 661-676.

Drechsler, Itamar and Amir Yaron (2011), "What's Vol Got to Do With It," Review of Financial Studies, vol. 24, 1-45. 